\title{
On dimension of porous measures
}

\author{
D. B. Beliaev • S. K. Smirnov
}

Received: 15 December 2000 / Published online: 1 February 2002 - C Springer-Verlag 2002

\begin{abstract}
We introduce a notion of mean porosity for measures and obtain dimensional bounds for mean-porous and porous measures. As a corollary we generalize to all porous Borel measures the estimate obtained recently by J.-P. Eckmann, E. Järvenpää, and M. Järvenpää for porous measures satisfying the doubling condition. We also discuss various generalizations of this notion and possible applications.
\end{abstract}

\section{Introduction}

The main purpose of this paper is to establish dimension and multifractal spectra estimates for porous measures. Dimensional properties of porous sets were extensively studied before: it is well known, that if every "piece" of a set in $\mathbb{R}^{d}$ has a "hole," its dimension admits an upper bound in terms of the relative size of the holes. It is hardly possible to obtain sharp estimates, so one is after the correct asymptotics when the holes are either big or small. If holes have size close to maximal, then the set must have dimension close to $d-1$, as was shown by P. Mattila in [Mat], and the correct asymptotics was found by A. Salli in [S]. For any porous set the dimension has to be strictly smaller than $d$. For a larger class of mean porous sets, the correct asymptotics of dimension estimate in terms of (small) size of holes and percentage of scales, having holes, was established by P. Koskela and S. Rohde in [KR].

Many measures, appearing as results of iterative constructions or dynamical considerations, are also porous (i.e. very nonuniformly distributed), thus it would be interesting to describe their dimensional properties. The first paper on this subject [EJJ] was written recently by J.-P. Eckmann, E. Järvenpää, and M. Järvenpää. They introduced a notion of porous measure, and proved a dimension estimate (similar to that of A. Salli) for porous measures, satisfying the doubling condition. We prove the same estimate for all Borel measures. We also introduce a more general class of mean porous measures and prove for them dimension estimates, which are analogues of estimates of Salli and Koskela-Rohde for sets. Along the way we generalize Salli's estimate to mean porous sets.

D.B. Beliaev, S.K. Smirnov

Department of Mathematics, Royal Institute of Technology, S-10044 Stockholm, Sweden (e-mail: beliaev@math.kth.se / stas@math.kth.se) 
First we informally present the definitions and our results. For detailed definitions and formulations (which become rather technical since dimension and porosity admit many flavors) see Sects. 2.2 and 2.3.

Porosity and dimension. We say that a measure $\mu$ is $\alpha$-porous if every ball $B_{2 R}$ has an " $\alpha$-hole", i.e. a ball $B_{\alpha R} \subset B_{2 R}$ such that $\mu\left(B_{\alpha R}\right) / \mu\left(B_{2 R}\right)=o(1)$, $R \rightarrow 0$. We introduce mean $(\alpha, \kappa)$-porous measures as those for which $\kappa \in$ $[0,1]$ proportion of exponentially shrinking concentric balls have " $\alpha$-holes." By $\operatorname{dim}_{p}(\mu)$ we denote the packing dimension of a measure $\mu$. For precise definitions see Sect. 2, possible generalizations are discussed in Sect. 6.

Theorem 1. There are positive constants $C_{1}$ and $C_{2}$ depending only on $d$ such that for any finite mean $(\alpha, \kappa)$-porous Borel measure $\mu$ on $\mathbb{R}^{d}$ the following estimates hold

$$
\begin{aligned}
& \operatorname{dim}_{p}(\mu) \leq d-\kappa+\frac{C_{1}}{|\log (1-\alpha)|}, \\
& \operatorname{dim}_{p}(\mu) \leq d-C_{2} \kappa \alpha^{d},
\end{aligned}
$$

particularly, if $\mu$ is $\alpha$-porous,

$$
\begin{aligned}
& \operatorname{dim}_{p}(\mu) \leq d-1+\frac{C_{1}}{|\log (1-\alpha)|}, \\
& \operatorname{dim}_{p}(\mu) \leq d-C_{2} \alpha^{d} .
\end{aligned}
$$

Remark 1. There are two definitions of porosity: upper and lower, and correspondingly one obtains estimates on upper and lower packing dimensions - see Sect. 5 for details. The third estimate above was obtained in [EJJ] for measures satisfying the doubling condition ${ }^{1}$. It is interesting when porosity is close to maximal, and has the correct order when $\alpha \nearrow 1$. Similarly the fourth estimate has the correct order when $\alpha \searrow 0$.

In deriving the Theorem 1, we will use the following estimates for Minkowski dimension of porous sets. The first is our generalization of Salli's estimate, and the second one is due to Koskela-Rohde [KR].

Theorem 2. There is a positive constant $C$ depending only on $d$ such that for any bounded uniformly mean ( $\alpha, \kappa)$-porous set $E \subset \mathbb{R}^{d}$ the following holds

$$
\overline{\operatorname{dim}_{M}}(E)<d-\kappa+\frac{C}{|\log (1-\alpha)|} .
$$

\footnotetext{
${ }^{1}$ When this paper was distributed, we received a new preprint [JJ] of E. Järvenpää and M. Järvenpää, where the estimate $\operatorname{dim}_{p}(\mu) \leq d-1+o(1), \alpha \rightarrow 1$ for general measures was proved by a method different from ours.
} 
Theorem 3 (Koskela, Rohde). There is a positive constant $C$ depending only on $d$ such that for any bounded uniformly mean $(\alpha, \kappa)$-porous set $E \subset \mathbb{R}^{d}$ the following holds

$$
\overline{\operatorname{dim}_{M}}(E)<d-C \kappa \alpha^{d} .
$$

In obtaining their estimate, J.-P. Eckmann, E. Järvenpää, and M. Järvenpää applied the following scheme: they have shown that for a porous measure satisfying the doubling condition there exists a porous set of positive measure, and then applied Salli's result. They have noted (see the Example 4 in [EJJ]) that the last statement might fail for non-doubling measures. We apply a similar approach, showing that every measure satisfies the "mean-doubling condition" (i.e. is doubling on most scales - see Sect.4), and deducing in the Proposition 1 that for a porous measure there exists a mean porous (with percentage of porous scales arbitrarily close to one) set of positive measure. Then we apply our generalization of Salli's result to mean porous sets (or Koskela-Rohde theorem for another estimate). The latter is proven by modification of Salli's methods and new inductive argument. The Proposition 1 seems to be of independent interest, in full generality it states that if a measure is mean porous, then there is subset of positive measure, which is mean porous with asymptotically the same percentage of porous scales.

Organization of the paper. In Sect. 2 we give precise definitions of mean-porous sets and measures and different definitions of a dimension of a measure. In the Sect. 3 we prove our generalization of Salli's theorem and sketch the proof of Koskela-Rohde's theorem. In Sect. 4 we prove that all Borel measures satisfy the "mean-doubling condition" and in Sect. 5 we prove the Theorem 1. In Sect. 6 we discuss possible generalizations of the porosity notions, extensions of our theorems, and perspective applications.

\section{Notation and definitions}

\subsection{Porosity of sets}

We denote by $B(x, r)$ a closed ball of radius $r$ centered at $x$ and by $B^{\circ}(x, r)$ a similar open ball. Let $E \subset \mathbb{R}^{n}$ be a bounded set and $0<\alpha, \rho<1$. We say that $E$ is $\alpha$-porous at point $x$ for scale $\log _{2}(1 / t)$ if there exists a point $y$ such that $B(y, \alpha t) \cap E=\emptyset$ and $|x-y|<t$. Throughout the proofs $\alpha$ will be fixed so we will write just porous without mentioning $\alpha$. For convenience we shall also say that $E$ is porous for scale $t$ but always when we write about some parts of scales we mean logarithmic scales.

Denote by $A_{n}(x)$ the annulus $A_{n}(x)=\left\{y \in \mathbb{R}^{n}: 2^{-n}<|x-y|<2^{-n+1}\right\}$. We say that there is a hole near $x$ for scale $n$ if there is a point $y$ in annulus $A_{n}(x)$ 
such that $E \cap B(y, \alpha|x-y|)=\emptyset$. We say that $E$ is $(\alpha, \rho)$-porous at a point $x$ if for sufficiently large $N$ at least $\rho N$ scales among scales $n=n_{0}(x)+1, \ldots, n_{0}(x)+N$ have a hole near $x$. Next, $E$ is mean $(\alpha, \rho)$-porous if it is mean $(\alpha, \rho)$-porous at every point $x \in E$.

If $n_{0}(x)$ are uniformly bounded from above, we say that $E$ is uniformly mean $(\alpha, \rho)$-porous.

\subsection{Porosity of measures}

We say that a Borel measure $\mu$ is mean $(\alpha, \kappa)$-porous at point $x$ if for all sufficiently small $\epsilon>0$ there exists a starting scale $r_{0}=r_{0}(\epsilon, x)$ such that for at least $\kappa N$ values of $r$ among $r_{0}, r_{0} 2^{-1}, \ldots, r_{0} 2^{-N+1}$ there exists a point $z$ such that $B(z, \alpha r) \subset B(x, 2 r)$ and $\mu(B(z, \alpha r)) \leq \epsilon \mu(B(x, 2 r))$. We say that $\mu$ is mean $(\alpha, \kappa)$-porous if it is $(\alpha, \kappa)$-porous at $\mu$-almost all points $x$.

If for every $\epsilon$ we can chose $r_{0}$ independently of $x$ then we say that measure $\mu$ is uniformly mean-porous.

The so defined porosity can be more precisely called upper porosity, to distinguish from lower porosity, when one requires condition above to hold for positive measure $\mu$ of points $x$.

\subsection{Dimension of measures}

Like porosity, dimension of a measure can be defined in several ways. One can calculate (various) dimensions of the sets of full (or big) measure or the sets of positive (may be small) measure.

One defines upper and lower packing dimensions of a measure (see [F, p. 171] for more detailed discussion) as

$$
\begin{aligned}
\left(\operatorname{dim}_{\mathrm{p}}\right)^{*}(\mu) & :=\lim _{\epsilon \rightarrow 0} \inf \left\{\operatorname{dim}_{\mathrm{p}}(E): \mu\left(E^{c}\right)<\epsilon\right\} \\
& =\inf \left\{\operatorname{dim}_{\mathrm{p}}(E): \mu\left(E^{c}\right)=0\right\}, \\
\left(\operatorname{dim}_{\mathrm{p}}\right)_{*}(\mu) & :=\inf \left\{\operatorname{dim}_{\mathrm{p}}(E): \mu(E)>0\right\} \leq\left(\operatorname{dim}_{\mathrm{p}}\right)^{*}(\mu)
\end{aligned}
$$

one can also define upper and lower Hausdorff dimensions in this way. Here and below we will use $\operatorname{dim}_{\mathrm{p}}$, $\operatorname{dim}_{\mathrm{H}}$, and $\overline{\operatorname{dim}}_{\mathrm{M}}$ to denote packing, Hausdorff, and upper Minkowski dimensions of a set (see [F, pp. 19-23]). We remind that always $\operatorname{dim}_{\mathrm{H}} E \leq \operatorname{dim}_{\mathrm{p}} E \leq \overline{\operatorname{dim}_{\mathrm{M}}} E$.

\section{Dimension of mean-porous sets}

First we note that the Theorems 2 and 3 admits the following corollary, since a mean porous set can be represented as a countable union of uniformly mean 
porous sets and $\operatorname{dim}_{\mathrm{p}}\left(\cup E_{i}\right)=\sup \operatorname{dim}_{\mathrm{p}}\left(E_{i}\right) \leq \sup \overline{\operatorname{dim}_{\mathrm{M}}}\left(E_{i}\right)$, we arrive at the following

Corollary 1. There are positive constants $C_{1}$ and $C_{2}$, depending on d only, such that for every bounded mean $(\alpha, \kappa)$-porous set $E \subset \mathbb{R}^{d}$ one has

$$
\begin{aligned}
& \operatorname{dim}_{\mathrm{p}}(E)<s=d-\kappa+\frac{C_{1}}{|\log (1-\alpha)|}, \\
& \operatorname{dim}_{\mathrm{p}}(E)<d-C_{2} \kappa \alpha^{d} .
\end{aligned}
$$

In [S] A. Salli proved a result similar to the Theorem 2 for porous sets which corresponds to taking $\kappa=1$ in our theorem (mean uniformly $(\alpha, 1)$-porous sets in our terminology are $\left((1+\alpha) / 2, r_{0}\right)$-porous in his). We use most of Salli's techniques adapting them to our situation. We reproduce without proofs two geometrical lemmas from [S] and prove one lemma which is a modification of the Lemma 3.3 from $[\mathrm{S}]$.

First we define some layers around sets. Let $E \subset \mathbb{R}^{n}$ and $r>0$. In what follows we shall denote

$$
\begin{aligned}
& M^{\circ}(E, r):=\left\{x \in \mathbb{R}^{n}: d(x, E)<r\right\}, \\
& M(E, r):=\left\{x \in \mathbb{R}^{n}: d(x, E) \leq r\right\} .
\end{aligned}
$$

Lemma 1. Write $C(s)=\left\{x \in \mathbb{R}^{d}: d(x, E) \geq s\right\}$ and $V(s)=M^{\circ}(C(s), s)$. If $E$ is porous at point $x$ for scale then

$$
x \in M(\partial V(\alpha t), t(1-\alpha)) .
$$

Proof. This lemma is a modification of the Lemma 3.3 in [S] and we use the same technique.

If $E$ is porous for scale $t$ at point $x$ then there is a point $y$ such that $B(y, \alpha t) \cap E$ $=\emptyset$ and $|x-y|<t$. This means that $y \in C(\alpha t)$ and $B^{\circ}(y, \alpha t) \subset V(\alpha t)$. Since $y$ is in $V(\alpha t)$ and $x$ is obviously not, the line segment from $x$ to $y$ contains some point of $\partial V(\alpha t)$, say $v$. Since $B^{\circ}(y, \alpha t) \subset V(\alpha t),|v-y| \geq \alpha t$ we have

$$
\mathrm{d}(x, \partial V(\alpha t)) \leq|x-v|=|x-y|-|y-v| \leq t-\alpha t=(1-\alpha) t,
$$

which proves the lemma.

Corollary 2. If $E$ is $\alpha$-porous for scale $r$ at a point $b$ then

$$
E \cap B(b, \beta r) \subset M(\partial V(r(1-2 \beta)), 2 \beta r) \cap B(b, \beta r),
$$

where $\beta=1-\alpha$.

Proof. It is easy to see that if $E$ is $\alpha$-porous for scale $r$ in point $b$ then $E$ is $(1-2 \beta)$ porous for scale $r$ in every point of $B(b, \beta r)$. Hence if $a \in B(b, \beta r) \cap E$ then $a \in M(\partial V(r(1-2 \beta)), 2 r \beta) \cap B(b, \beta r)$. So

$$
E \cap B(b, \beta r) \subset M(\partial V(r(1-2 \beta) r), 2 r \beta) \cap B(b, \beta r) .
$$


Lemma 2. Let $V=M^{\circ}(C, r)$ where $C \subset \mathbb{R}^{d}$ and $r>0$. Let $\epsilon>0$ and $b \in \mathbb{R}^{d}$ be arbitrary. Then there is a compact convex set $K \subset B(b, \epsilon r)$ such that

$$
M\left(\partial V, \epsilon^{2} r\right) \cap B(b, \epsilon r) \subset M\left(\partial K, 3 \epsilon^{2} r\right) \cap K .
$$

Proof. See Lemma 2.6 in $[\mathrm{S}]$.

Lemma 3. Let $a \in \mathbb{R}^{d}, r>0,0<\delta<1$, and suppose that $K \subset B(a, r)$ is $a$ convex set. Then

$$
|M(\partial K, \delta r)| \leq c \delta|B(a, r)|
$$

where $c=c(n)<\infty$ is a constant.

Proof. See Lemma 2.4 in [S].

Proof (Theorem 2). Let $\beta=1-\alpha$. Let $E$ be porous for scale $r$ at point $b$. By the Corollary to the Lemma $1 E \cap B(b, \beta r) \subset M(\partial V(r(1-2 \beta)), 2 \beta r) \cap B(b, \beta r)$. Denote $r(1-2 \beta)$ by $t$ and $\beta /(1-2 \beta)$ by $\epsilon^{2}$ and rewrite previous inclusion using new notation

$$
E \cap B\left(b, \epsilon^{2} t\right) \subset M\left(\partial V(t), 2 \epsilon^{2} t\right) \cap B\left(b, \epsilon^{2} t\right) .
$$

If $\beta$ is sufficiently small then $\epsilon^{2}<\epsilon \sqrt{2}$ hence by the Lemma 2

$$
\begin{aligned}
M\left(\partial V(t), 2 \epsilon^{2} t\right) \cap B\left(b, \epsilon^{2} t\right) & \subset M\left(\partial V(t), 2 \epsilon^{2} t\right) \cap B(b, \epsilon \sqrt{2} t) \\
& \subset M\left(\partial K, 6 \epsilon^{2} t\right) \cap K
\end{aligned}
$$

where $K \subset B(b, \epsilon \sqrt{2} t)$. So we have $E \cap B\left(b, \epsilon^{2} t\right) \subset M\left(\partial K, 6 \epsilon^{2} t\right)$ and by the Lemma 3

$$
\left|M\left(E \cap B\left(b, \epsilon^{2} t\right), \epsilon^{2} t\right)\right| \leq\left|M\left(\partial K, 7 \epsilon^{2} t\right)\right| \leq c \frac{7 \epsilon^{2}}{\epsilon \sqrt{2}}|B(b, \epsilon \sqrt{2} t)| .
$$

Let $Q(b, r)$ be a cube with side length $r$ and with faces parallel to coordinate planes.

Note that if intersection in the left hand side of (1) is not empty then we can change balls to cubes

$$
\left|M\left(E \cap Q\left(b, \frac{\epsilon^{2} t}{\sqrt{n}}\right), \frac{\epsilon^{2} t}{\sqrt{n}}\right)\right| \leq C \epsilon\left|M\left(E \cap Q\left(b, \frac{\epsilon^{2} t}{\sqrt{n}}\right), \frac{\epsilon t}{n^{1 / 4}}\right)\right| .
$$

So taking new $\epsilon=\sqrt{\beta /((1-2 \beta) \sqrt{n})}$ we obtain

$$
\left|M\left(E \cap B\left(b, \epsilon^{2} t\right), \epsilon^{2} t\right)\right| \leq C \epsilon\left|M\left(E \cap B\left(b, \epsilon^{2} t\right), \epsilon t\right)\right| .
$$

We write $Q \in$ "Porous" if $E$ is porous for scale $r \epsilon^{-2}$ in point $b$ where $Q$ is a cube, $r$ is the side length of $Q$, and $b$ is the center of $Q$. 
Take small $\epsilon$ with $\epsilon^{-1} \in \mathbb{N}$. Divide space into cubes with side length $\epsilon r$, after that we divide each cube into $\epsilon^{n}$ cubes with side length $\epsilon^{2} r$, and so on. Let $Q_{1}$ and $Q_{2}$ be two cubes from this grid. We shall write $Q_{1}<Q_{2}$ if $Q_{1} \subset Q_{2}$ and the side length of $Q_{1}$ is $\epsilon$ times shorter then the side length of $Q_{2}$. We denote by $l(Q)$ the side length of $Q$.

Obviously $\cup_{Q^{\prime}<Q} M\left(E \cap Q^{\prime}, l\left(Q^{\prime}\right)\right)=M(E \cap Q, \epsilon l(Q))$ and every point of the set on the right hand side is covered by the sets on the left hand side no more then $c(d)$ times. So we have an estimate on measures

$$
\begin{aligned}
\mid M(E \cap Q, \epsilon l(Q) \mid & \leq \sum_{Q^{\prime}<Q}\left|M\left(E \cap Q^{\prime}, l\left(Q^{\prime}\right)\right)\right| \\
& \leq c(n)|M(E \cap Q, \epsilon l(Q))| .
\end{aligned}
$$

Combining this inequality with (2) we get for "Porous" $Q$

$$
\left|M\left(E \cap Q(b, \epsilon r), \epsilon^{2} r\right)\right| \leq c \epsilon|M(E \cap Q(b, \epsilon r), \epsilon r)|,
$$

and by inclusion it is obvious that for every $Q$

$$
\left|M\left(E \cap Q(b, \epsilon r), \epsilon^{2} r\right)\right| \leq|M(E \cap Q(b, \epsilon r), \epsilon r)| .
$$

Combining (3) with (4) and (5) we get

$$
\sum_{Q^{\prime}<Q}\left|M\left(E \cap Q^{\prime}, l\left(Q^{\prime}\right)\right)\right|<\left\{\begin{array}{l}
C|M(E \cap Q, l(Q))| Q \notin \text { "Porous", } \\
C \epsilon|M(E \cap Q, l(Q))| Q \in \text { "Porous". }
\end{array}\right.
$$

To simplify the following we shall abbreviate

$$
\begin{aligned}
& |M(E \cap Q, l(Q))|=\beta(Q), \\
& \gamma(Q)= \begin{cases}1 & l(Q)=\epsilon r \\
C & Q \notin \text { "Porous", } \\
C \epsilon & Q \in \text { "Porous" }\end{cases} \\
& \alpha(Q)=\prod_{j} \gamma\left(Q_{j}\right) \quad \text { where } Q<Q_{k}<\cdots<Q_{1}<Q_{0} .
\end{aligned}
$$

Note that the constant $C$ depends on dimension only and is independent of $\epsilon$.

Rewriting (6) with new notations we get

$$
\sum_{Q^{\prime}<Q} \frac{\beta\left(Q^{\prime}\right)}{\alpha\left(Q^{\prime}\right)} \leq \frac{\beta(Q)}{\alpha(Q)}
$$

Combining this inequality for all "descendants" of $k$-th generation of a cube $Q_{0}$ with side length $\epsilon r$ we obtain

$$
\sum_{l(Q)=\epsilon^{k+1} r, Q \subset Q_{0}} \frac{\beta(Q)}{\alpha(Q)} \leq \beta\left(Q_{0}\right) .
$$


Take $\beta_{1}=3 \beta$ then if set $E$ is $(1-\beta)$-porous for scale $n$ then $E$ is $\left(1-\beta_{1}\right)$ porous for all scales $t \in\left[2^{-n-1}, 2^{-n}\right]$. We can write all previous estimates for $\beta_{1}$. Fix any point $b \in E$ and denote by $G_{N}=G_{N}(b)$ the set

$$
\begin{array}{r}
\left\{w: \log _{1 / 2} r \leq w \leq N+\log _{1 / 2} r, E \text { is }(1-3 \beta)-\right.\text { porous } \\
\text { for scale } w \text { at point } b\} .
\end{array}
$$

Since $E$ is mean $(\alpha, \kappa)$-porous, length $\left(G_{N}\right)>\kappa N$ for all $r$ if $N$ is sufficiently large.

We want to estimate $\alpha(Q)$ for a given small cube with side length $\epsilon^{k+1} r$. Let $b$ be center of $Q$ and $l(Q)=\epsilon^{k+1} r$ so $Q$ is a cube of $k$-th generation. We want to know how many scales among $\epsilon^{k} r, \ldots, r$ are $(1-3 \beta)$-porous.

Note that we had no assumptions about $r$. If we change $r$ to $r 2^{-j}$ we just shift our selected scales. It is easy to see that for every point $b$ there exists $j<\log _{1 / 2} \epsilon$ such that if we take $r_{0}(N)=r 2^{-j} \epsilon$ as the size of the first cube then at least $\kappa(k-1)$ among scales $\epsilon^{k} r_{1}, \ldots, r_{1}$ will be $(1-3 \beta)$-porous. So if $r=r_{0}(N)$ then $\alpha(Q) \leq C^{k-1} \epsilon^{\kappa(k-1)}$ hence

$$
\begin{aligned}
\beta(Q) & \leq \beta\left(Q\left(b, \epsilon^{k} r_{0}(k)\right)\right) \leq C^{(k-1)} \epsilon^{\kappa(k-1)} \\
& \leq C^{(k-1)} \epsilon^{\kappa(k-1)} \beta\left(Q\left(b, \epsilon r_{0}(k)\right)\right) \leq C^{(k-1)} \epsilon^{\kappa(k-1)} \beta\left(Q_{0}\right) .
\end{aligned}
$$

Finally combining this estimate with (7) we obtain

$$
C^{(k-1)} \epsilon^{\kappa(k-1)} \beta\left(Q_{0}\right) \geq \sum_{l(Q)=\epsilon^{k+1} r, Q \subset Q_{0}} \beta(Q) \geq\left|M\left(E \cap Q_{0}, \epsilon^{k+1} r\right)\right| .
$$

Summing over all such $Q_{0}$ we obtain

$$
\left|M\left(E, \epsilon^{k+1} r\right)\right| \leq\left(C \epsilon^{\kappa}\right)^{(k-1)}|M(E, \epsilon r)| \quad \text { for all sufficiently large } k .
$$

For an arbitrary small $t$ let $k$ be such that $t \in\left(\epsilon^{k+2} r, \epsilon^{k+1} r\right]$ then $|M(E, t)|<$ $\left|M\left(E, \epsilon^{k+1} r\right)\right|$. Hence by $(8)$

$$
|M(E, t)| \leq\left(C \epsilon^{\kappa}\right)^{(k-1)}|M(E, \epsilon r)| .
$$

Write

$$
s=d-\kappa+\log (C) /|\log \epsilon|<d-\kappa+C_{0} /|\log \beta|=s^{\prime}
$$

so that $\epsilon^{d-s}=C \epsilon^{\kappa}$ this gives

$$
\left(C \epsilon^{\kappa}\right)^{(k-1)}=\epsilon^{(k-1)(d-s)}<\left(\frac{t}{\epsilon^{3} r}\right)^{d-s}<C_{1} t^{d-s}<C_{1} t^{d-s^{\prime}} .
$$

Combining this with (9) we obtain

$$
|M(E, t)| \leq C_{1}|M(E, \epsilon r)| t^{d-s^{\prime}}=\text { const } t^{d-s^{\prime}} .
$$


It is a well known fact that $(10)$ implies $\overline{\operatorname{dim}_{M}}(E) \leq s^{\prime}$. For reader's convenience we sketch the proof.

Since the $\epsilon$-cubes contained in the $\sqrt{n} \epsilon$-neighborhood of $E$ cover $E$ completely, one can write

$$
N(E, \epsilon) \leq \epsilon^{-n}|M(E, \sqrt{n} \epsilon)|
$$

where $N(E, \epsilon)$ is the number of $\epsilon$-cubes needed to cover $E$. Thus

$$
\overline{\operatorname{dim}_{\mathrm{M}}}(E)=\lim _{\epsilon \rightarrow 0} \sup \frac{\log N(E, \epsilon)}{|\log \epsilon|} \leq s^{\prime} .
$$

Proof ( Theorem 3). This theorem was proved in [KR] with $\kappa=1 / 2$, and we sketch modification of their proof for arbitrary $\kappa$.

First we claim that there is a constant $c$ depending on dimension $d$ only such that for any porous $E$ there exists a family $\mathcal{B}$ of pairwise disjoint open balls $B \subset$ $M(E, 1)$ with the property that for every integer $j$ and every $x \in M\left(E, 2^{-j}\right)$,

$$
\sum_{B \in \mathcal{B}} \chi_{\left(\frac{c}{\alpha}\right) B}(x) \geq c_{1} \kappa j .
$$

To prove the claim consider a Whitney decomposition of $\mathbb{R}^{d} \backslash E$. Divide every Whitney cube into $M^{d}$ cubes ( $M$ is a large constant depending only on $d$ ). Let $\mathcal{B}$ be a family of balls inscribed into these cubes. If there is a hole near point $x \in E$ for scale $n$ then there exists $B=B(y, r) \in \mathcal{B}$ such that $B \subset A_{n}(x)$ and $B\left(x, 2^{-j}\right) \subset B(y, c r / \alpha)$. Since $E$ is mean $(\alpha, \kappa)$-porous we obtain the claim.

To prove the Theorem we will use the following inequality which is a well known consequence of the Hardy-Littlewood theorem:

If $\mathcal{B}$ is a family of balls in $\mathbb{R}^{d}$ and $p>0$, then

$$
\int_{\mathbb{R}^{d}}\left(\sum_{B \in \mathcal{B}} \chi_{B / p}(x)\right)^{k} d x \leq\left(\frac{C_{1} k}{p^{d}}\right)^{k} \int_{\mathbb{R}^{d}}\left(\sum_{B \in \mathcal{B}} \chi_{B}(x)\right)^{k} d x
$$

for all $k \geq 1$, where $C_{1}=C_{1}(d)$.

We want to prove that

$$
\left|M\left(E, 2^{-j}\right)\right| \leq c_{1}|M(E, 1)| 2^{-c_{2} j \kappa \alpha^{d}} .
$$

for all $j \geq 1$ with constants $c_{1}$ and $c_{2}$ depending on $d$ only. It is a well known fact that this inequality implies the desired estimate of dimension.

It is sufficient to show that for some constant $\gamma$

$$
\int_{M\left(E, 2^{-j}\right)} \exp \left(\gamma \alpha^{d} \sum_{B \in \mathcal{B}} \chi_{\left(\frac{c}{\alpha}\right) B}(x) d x\right) \leq c_{2}|M(E, 1)|
$$


because

$$
\exp \left(\gamma \alpha^{d} \sum \chi_{\left(\frac{c}{\alpha}\right) B}(x)\right) \geq \exp \left(\gamma \kappa c_{1} j \alpha^{d}\right)
$$

on $M\left(E, 2^{-j}\right)$.

Write

$$
u(x)=\gamma \alpha^{d} \sum_{B \in \mathcal{B}} \chi_{\left(\frac{c}{\alpha}\right) B}(x) .
$$

Then

$$
\begin{aligned}
\int_{M\left(E, 2^{-j}\right)} & \exp (u(x)) d x \leq \\
& \leq \int_{M(E, 1)} \exp (u(x)) d x \leq \sum_{k \geq 1} \int_{M(E, 1)} \frac{u(x)^{k}}{k !} d x \\
& \leq|M(E, 1)|+\sum_{k \geq 1} \int_{\mathbb{R}^{d}} \frac{u(x)^{k}}{k !} d x \\
& \leq|M(E, 1)|+\sum_{k \geq 1} \frac{\left(\gamma \alpha^{d}\right)^{k}}{k !} \int_{\mathbb{R}^{d}} \sum_{B \in \mathcal{B}}\left(\chi_{\left(\frac{c}{\alpha}\right) B}(x)\right)^{k} d x .
\end{aligned}
$$

Using (12) we obtain

$$
\begin{aligned}
\exp (u(x)) d x & \leq|M(E, 1)|+\sum_{k \geq 1} \frac{\left(\gamma \alpha^{d}\right)^{k}}{k !}\left(\frac{C_{1} k}{\alpha^{d}}\right)^{k} \int_{\mathbb{R}^{d}} \sum_{B \in \mathcal{B}}\left(\chi_{B}(x)\right)^{k} d x \\
& \leq|M(E, 1)|\left(1+\sum_{k>0} \frac{\left(C_{1} \gamma k\right)^{k}}{k !}\right) .
\end{aligned}
$$

Since the last series converges for sufficiently small $\gamma$ we have obtained the desired estimate and finished the proof of Theorem.

\section{Mean-doubling condition}

In this section we prove that any Borel measure satisfies the doubling condition on almost all scales at all points outside a small exceptional set.

Let $\mu$ be a finite Borel measure supported on a bounded set in $\mathbb{R}^{d}, z$ be any point, $k$ be a large number, and $i \in[1, \ldots, n]$. For convenience we suppose that $\mu$ is supported in the unit ball and that full measure is equal to 1 .

Fix a large constant $C$ and small $\rho$. Denote by $E_{n}$ the set of points $z$ such that for more then $\rho n$ values of $i \in[1, \ldots, n]$ we have $C \mu\left(B\left(z, k^{-i}\right)\right)<$ 
$\mu\left(B\left(z, k^{-i+1}\right)\right)$. Then $\mu\left(B\left(z, k^{-n}\right)\right) \leq C^{-n \rho}$. Cover support of $\mu$ by $2^{d} k^{n d}$ balls of radius $k^{-n}$ such that every point is covered no more than $c(d)$ times. The measure of the union of balls satisfying $C \mu\left(B\left(z, k^{-n}\right)\right)<C^{-n \rho}$ is no more than $\left(2^{d / n} k^{d} C^{-\rho}\right)^{n}$. So $\mu\left(E_{n}\right)<c(d) 2^{d}\left(k^{d} C^{-\rho}\right)^{n}$. Denoting $\cup_{n=1}^{\infty} E_{n}$ by $E_{C}$ we can write

$$
\mu\left(E_{C}\right) \leq \sum_{n=1}^{\infty} c(d) 2^{d}\left(k^{d} C^{-\rho}\right)^{n}=\frac{c(d) 2^{d} Q}{1-Q},
$$

where $Q=k^{d} C^{-\rho}$.

What is $\mathbb{R}^{d} \backslash E_{C}$ ? It is the set of points $z$ such that for all $n>1$ the number of $i \in[1, \ldots n]$ with $C \mu\left(B\left(z, k^{-i}\right)\right)>\mu\left(B\left(z, k^{-i+1}\right)\right)$ is at least $(1-\rho) n$. If $k$ and $\rho$ are given we can chose $C$ large enough to make $\mu\left(E_{C}\right)$ as small as we want.

\section{Dimension of porous measures}

Proposition 1. Let $\mu$ be a finite mean $(\alpha, \kappa)$-porous Borel measure on $\mathbb{R}^{d}$. Then for any positive $\delta$ and $\sigma$ there is a set $B$ such that $\mu\left(B^{c}\right)<\sigma$ and $B$ is mean $(\alpha-\delta, \kappa-\delta)$-porous. If $\mu$ is lower mean porous then there is a set $B$ such that $\mu(B)>0$ and $B$ is mean $(\alpha-\delta, \kappa-\delta)$-porous.

Proof. For convenience we can assume that $\mu$ is supported in unit cube and that full measure is 1 . Fix small $\sigma>0$. Let $A=\{x \in \operatorname{spt}(\mu): \mu$ is mean $(\alpha-\delta, \kappa)$ porous at $x$ \} where $\delta>0$. If $\delta$ is sufficiently small then $\mu(A)>1-\sigma / 2$. Take $k=4 \delta^{-1}$ and $\rho=\delta$. Then by (14) we can chose $C$ so large that $\mu\left(E_{C}\right)<\sigma / 2$. Write $B=A \backslash E_{C}$ then $\mu(B)>1-\sigma$. We want to prove that $B$ is mean $(\alpha-2 \delta, \kappa-\rho)$-porous.

Let $x$ be any point of $B$, by definition of $A$ for sufficiently small $\epsilon$ and $r_{0}$ there exists point $z$ such that $B(z,(\alpha-\delta) r) \subset B(x, 2 r)$ and $\mu(B(z,(\alpha-\delta) r)) \leq$ $\epsilon \mu(B(x, 2 r))$ for at least $\kappa N$ values of $r=r_{0}, \ldots, r_{0} 2^{-N+1}$. We will prove that

$$
B(z,(\alpha-2 \delta) r) \cap B=\emptyset
$$

for at least $(\kappa-\delta) N$ among $N$ scales. So we will prove that $B$ is mean $(\alpha-$ $2 \delta, \kappa-\delta)$-porous.

To prove (15), we assume that there exists $y \in B(z,(\alpha-2 \delta) r) \cap B$. Then

$$
\mu(B(y, \delta r)) \leq \mu(B(z,(\alpha-\delta) r)) \leq \epsilon \mu(B(x, 2 r)) \leq \epsilon \mu(B(y, 4 r)) .
$$

By definition of $E_{C}$ if $N$ is large enough then for $(1-\delta) N$ scales among $N$ scales starting from scale $r(x)$ the right hand side is less then $C \epsilon \mu(B(y, \delta r))$ what leads us to contradiction since we can chose $\epsilon$ very small.

If $\mu$ is lower porous then similar argument gives $B$ of positive measure. 
Theorem 1. There are positive constants $C_{1}$ and $C_{2}$ depending only on $d$ such that for any finite mean $(\alpha, \kappa)$-porous Borel measure $\mu$ on $\mathbb{R}^{d}$ the following holds

$$
\begin{aligned}
& \left(\operatorname{dim}_{p}\right)^{*}(\mu) \leq d-\kappa+\frac{C_{1}}{|\log (1-\alpha)|}, \\
& \left(\operatorname{dim}_{p}\right)^{*}(\mu) \leq d-C_{2} \kappa \alpha^{d},
\end{aligned}
$$

particularly, if $\mu$ is $\alpha$-porous,

$$
\begin{aligned}
& \left(\operatorname{dim}_{p}\right)^{*}(\mu) \leq d-1+\frac{C_{1}}{|\log (1-\alpha)|}, \\
& \left(\operatorname{dim}_{p}\right)^{*}(\mu) \leq d-C_{2} \alpha^{d} .
\end{aligned}
$$

Proof. Fix small $\delta$ and $\sigma$ then by the Proposition 1 there is a set $B$ such that $\mu\left(B^{c}\right)<\sigma$ and $B$ is mean $(\alpha-\delta, \kappa-\delta)$-porous. By the Corollary 1

$$
\begin{aligned}
& \inf \left\{\operatorname{dim}_{\mathrm{p}}(E): \mu(E)>1-\sigma\right\}<d-\kappa+\delta+C_{1} /|\log (1-\alpha+\delta)|, \\
& \inf \left\{\operatorname{dim}_{\mathrm{p}}(E): \mu(E)>1-\sigma\right\}<d-C_{2}(\kappa-\delta)(\alpha-\delta)^{d}
\end{aligned}
$$

since $\delta$ and $\sigma$ can be arbitrary small we obtain desired estimate on dimension of measure.

Remark 2. If $\mu$ is lower mean porous then the same estimates hold for lower packing dimension $\left(\operatorname{dim}_{\mathrm{p}}\right)_{*}$.

Corollary 3. Let $\mu$ be a finite lower mean $(\alpha, \kappa)$-porous Borel measure on $\mathbb{R}^{d}$. Then there are positive constants $C_{1}$ and $C_{2}$ depending only on $d$ such that the following holds

$$
\begin{aligned}
& \left(\operatorname{dim}_{p}\right)_{*}(\mu) \leq d-\kappa+\frac{C_{1}}{|\log (1-\alpha)|}, \\
& \left(\operatorname{dim}_{p}\right)_{*}(\mu) \leq d-C_{2} \kappa \alpha^{d},
\end{aligned}
$$

particularly, if $\mu$ is lower $\alpha$-porous,

$$
\begin{aligned}
& \left(\operatorname{dim}_{p}\right)_{*}(\mu) \leq d-1+\frac{C_{1}}{|\log (1-\alpha)|}, \\
& \left(\operatorname{dim}_{p}\right)_{*}(\mu) \leq d-C_{2} \alpha^{d} .
\end{aligned}
$$

Remark 3. For a uniformly mean porous measures we can find a uniformly mean porous set with arbitrary large (or positive if we consider lower porosity) measure. So we obtain the same estimates for

$$
\left(\operatorname{dim}_{M}\right)^{*}(\mu)=\lim _{\epsilon \rightarrow 0} \inf \left\{\overline{\operatorname{dim}_{M}}(E): \mu\left(E^{c}\right)<\epsilon\right\}
$$


and (in the case of lower porosity)

$$
\left(\operatorname{dim}_{\mathrm{M}}\right)_{*}(\mu)=\inf \left\{\overline{\operatorname{dim}_{\mathrm{M}}}(E): \mu\left(E^{c}\right)>0\right\} .
$$

Upper Minkowski measure is not countably additive so upper Minkowski dimension for measures admits two a priori different definitions:

$$
\lim _{\epsilon \rightarrow 0} \inf \left\{\overline{\operatorname{dim}_{\mathrm{M}}}(E): \mu\left(E^{c}\right)<\epsilon\right\} \neq \inf \left\{\overline{\operatorname{dim}_{\mathrm{M}}}(E): \mu\left(E^{c}\right)=0\right\} .
$$

The following example shows that corresponding estimate for the second quantity does not hold.

Example 1. Let measure $\mu$ be a sum of $\delta$-measures:

$$
\mu=\sum_{n=1}^{\infty} \frac{1}{n^{n}} \delta_{\frac{1}{\log n}} .
$$

Then $\mu$ is uniformly 1-porous and $\lim _{\epsilon \rightarrow 0} \inf \left\{\overline{\operatorname{dim}_{M}}(E): \mu\left(E^{c}\right)<\epsilon\right\}=0$, while inf $\left\{\overline{\operatorname{dim}_{\mathrm{M}}}(E): \mu\left(E^{c}\right)=0\right\}=1$.

\section{Appendix: On the definition of porosity}

Porosity of measure as defined above is a very strong condition: we restrict ourselves to measures, dimension of whose compact support is strictly smaller than the dimension of the space. There are many naturally arising measures, which do not fall into this category, but whose dimension is nevertheless strictly smaller than the dimension of the space since they are "unevenly distributed."

Two important categories not completely covered by our definitions are Gibbs measures for dynamical systems and harmonic measures for domains in $\mathbb{R}^{d}$.

\subsection{Gibbs measures and weak porosity}

If we divide a cube into $k^{d}$ cubes with equal side length then their Lebesgue measures would be equal. If a measure $\mu$ is absolutely continuous with respect to Lebesgue measure then almost everywhere similar property holds asymptotically at small scales.

If a measure is required to violate such property then we can expect it to be very singular, perhaps with dimension strictly smaller than $d$.

We formulate this principle as follows: Borel measure $\mu$ in $\mathbb{R}^{d}$ is $(1 / k, \epsilon)$ weak porous if for any $k$-adic cube $Q$ there is a $k$-adic cube $Q^{\prime} \subset Q$ with $k$ times smaller side length such that $\mu\left(Q^{\prime}\right)<\epsilon \mu(Q) / k^{d}$.

This means that there is a subcube that carries smaller proportion of measure than it would for uniform distribution. 
The definition can also be relaxed by introducing "mean" version or requiring the property to hold only around almost all points and only for sufficiently small cubes. If $\epsilon$ is small, this definition is equivalent (up to a multiplicative change in constants) to a similar definition involving balls.

The condition above is interesting since it is weaker than the usual condition for porosity, but one still can obtain estimates for dimension and multifractal spectra of such measures.

Theorem 4. There exist a positive constant $C$ and a positive function $A(\epsilon, k)$ depending on d only, such that for any finite $(1 / k, \epsilon)$-weak porous Borel measure $\mu$ on $\mathbb{R}^{d}$ the following holds

$$
\operatorname{dim}_{\mathrm{H}}(\mu)<d-A(\epsilon, k) .
$$

Moreover

$$
A(\epsilon, k) \nearrow \frac{\log \left(\frac{k^{d}}{k^{d}-1}\right)}{\log k} \quad \text { as } \quad \epsilon \searrow 0,
$$

and

$$
A(\epsilon, k) \geq(1-\epsilon)^{2} \frac{2}{\left(k^{d}-1\right) \log k},
$$

when $\epsilon$ is close to 1 .

Proof. We will estimate multifractal spectra, see [F, chapter 11] and [Mak], which are continua of parameters comprehensively characterizing fine structure of measures (particularly they determine the dimension). We will estimate the packing spectrum $\pi_{\mu}(t)$ of the measure $\mu$ which is defined by

$$
\begin{array}{r}
\pi_{\mu}(t)=\sup \{q: \text { for all } \delta>0 \text { there is a } \delta-\operatorname{packing}\{B\} \\
\text { such that } \left.\sum \operatorname{diam}(B)^{t} \mu(B)^{q}>1\right\},
\end{array}
$$

where $\delta$-packing is the collection of disjoint balls with diameters less than $\delta$.

We will follow the so called "fractal approximation" principle, which says that in such problems extremal objects are self-similar.

We start with a weakly porous measure $\mu$ and show that it can be modified to be self-similar (Cantor-type) weakly porous measure $v$ with larger spectrum $\sigma:=\pi_{\nu} \geq \pi_{\mu}$. The spectrum $\sigma$ will be the solution of some specific functional equation.

First note that applying a Besicovitch covering argument, we can use in the definition of the packing spectrum $k$-adic cubes instead of balls. Observe also that if there is a $\delta$-packing (of cubes) such that $\sum l(Q)^{t} \mu(Q)^{q}>1$ then there is a finite $\delta$-packing with the same property. Finally note that if we take any $k$-adic cube from this packing and divide it into subcubes then $\sum \cdots$ will increase. Thus we can reduce this $\delta$-packing to one with equal sized cubes and $\sum \cdots>1$. 
This argument shows that one can equivalently define $\pi_{\mu}$ by

$$
\pi_{\mu}(t):=\lim _{n \rightarrow \infty} \pi_{\mu}(t, n),
$$

where $\pi_{\mu}(t, n)$ is the "spectrum at scale $n: "$

$$
\sup \left\{q: \sum l(Q)^{t} \mu(Q)^{q}>1\right\} \equiv \sup \left\{q: k^{-d t} \sum \mu(Q)^{q}>1\right\},
$$

where the sum is taken over all $k$-adic cubes with the side length $k^{-n}$.

Denote by $C_{n}(Q)$ the collection of $k^{\text {nd }}$ disjoint subcubes of $Q$ with the side length $k^{n}$ times smaller than the side length of $Q$. Set $\mu_{0}:=\mu$.

We construct inductively a sequence of measures $\mu_{l}$, preserving porosity and increasing spectrum. When $\mu_{l-1}$ is constructed, we rearrange it at scale $l$ to obtain the next measure $\mu_{l}$. Namely for every $Q \in C_{l-1}\left(Q_{0}\right)$ we choose some $Q^{\prime} \in C_{1}(Q)$ with $\mu_{l-1}\left(Q^{\prime}\right)<\epsilon k^{-d} \mu_{l}(Q)$. Such $Q^{\prime}$ exists because $\mu_{l-1}$ is porous. Then we define $\mu_{l}$ on $Q$ setting

$$
d \mu_{l}(x):=d \mu_{l-1}(x) \cdot \frac{\lambda\left(Q^{\prime \prime}\right)}{\mu_{l-1}\left(Q^{\prime \prime}\right)} \quad \text { for } x \in Q^{\prime \prime} \in C_{1}(Q),
$$

where

$$
\lambda\left(Q^{\prime \prime}\right):=\frac{k^{d}-\epsilon}{k^{d}-1} \quad \text { if } \quad Q^{\prime \prime} \neq Q^{\prime}, \quad \text { and } \lambda\left(Q^{\prime}\right):=\epsilon .
$$

Porosity is clearly preserved by this procedure. It is also easy to see that

$$
\pi_{\mu_{l}}(t, n)=\pi_{\mu_{l-1}}(t, n) \text { for } n<l,
$$

and by concavity of $x^{q}, q \leq 1$ (which is the only relevant case, since $\pi(t) \leq 1$ )

$$
\pi_{\mu_{l}}(t, n) \geq \pi_{\mu_{l-1}}(t, n) \text { for } n \geq l .
$$

Thus spectra $\pi(t, n)$ can only increase with $l$.

Our measures $\mu_{n}$ converge to a "Cantor-type" measure $v$ which can be described in the following way: for every $m \leq n$ exactly $\left(\begin{array}{l}n \\ m\end{array}\right)\left(k^{d}-1\right)^{m}$ of cubes in $C_{n}\left(Q_{0}\right)$ have measure $q^{m} p^{n-m}$ where

$$
\begin{aligned}
& q=\frac{k^{d}-\epsilon}{k^{d}\left(k^{d}-1\right)}, \\
& p=\frac{\epsilon}{k^{d}} .
\end{aligned}
$$

This description does not define $v$ uniquely, but all such measures have the same spectrum. Moreover, for every $n$

$$
\pi_{v}(t, n)=\left.\pi_{\mu_{l}}(t, n)\right|_{l \geq n} \geq \pi_{\mu}(t, n),
$$


so we infer that

$$
\sigma(t)=\lim _{n \rightarrow \infty} \pi_{\nu}(t, n) \geq \pi_{\mu}(t)
$$

Using the description of measure $v$ it is easy to estimate its spectrum at a given scale:

$$
\begin{aligned}
& \sum_{m=0}^{n} \operatorname{diam}\left(Q_{j}\right)^{t} v\left(Q_{j}\right)^{a}=k^{-n t} \sum_{m=0}^{n}\left(\begin{array}{l}
n \\
m
\end{array}\right)\left(q^{a}\left(k^{d}-1\right)\right)^{m} p^{a(n-m)} \\
& =k^{-n t}\left(q^{a}\left(k^{d}-1\right)+p^{a}\right)^{n},
\end{aligned}
$$

which gives us an equation describing $\sigma(t)$

$$
t=\frac{\log \left(q^{\sigma(t)}\left(k^{d}-1\right)+p^{\sigma(t)}\right)}{\log k} .
$$

It is then not difficult to see that $A(\epsilon, k):=d-1 /\left|\sigma^{\prime}(0)\right|$ satisfies asymptotics of Theorem 4. For any measure $\operatorname{dim}_{\mathrm{H}} \mu=1 /\left|\pi_{\mu}^{\prime}(0+)\right|$ (for further details see [F, 11] and [Mak, p. 226]), so (16) holds since both $\pi(t)$ and $\sigma(t)$ are convex functions and hence $\left|\pi_{\mu}^{\prime}(0+)\right| \geq\left|\sigma^{\prime}(0+)\right|$. Estimates of $A(\epsilon, k)$ we obtain by estimating $\sigma^{\prime}(0)$.

Our motivation for the definition of weak porosity comes from dynamical systems, where many relevant measures appear to satisfy it (and sometimes even the stronger definition). Under fairly general conditions one can expect the following dichotomy:

Dichotomy A Gibbs measure is either absolutely continuous, or weakly porous (and hence satisfies dimension and spectra estimates of the Theorem 4).

This (unrigorous) principle can be proved in many situations, and we sketch its proof for the dynamical system $T: x \mapsto k x \bmod 1$ on the unit interval $I_{0}$.

Indeed, assume that $\mu$ is a Gibbs measure, i.e. it is associated to some Hölder potential $\phi$ (for this and other facts see [Bow]). Denote by $P$ the pressure of $\phi$ and set $S_{n} \phi(x):=\sum_{j=0}^{n-1} \phi\left(T^{j} x\right)$. Then Bowen's formula says that whenever $|x-y| \leq k^{-n}$, one can estimate the Jacobian of the measure $\mu$ with respect to the iterate $T^{n}$ of the map $T$ by

$$
\frac{d \mu(x)}{d \mu\left(T^{n} x\right)} \asymp \exp \left(S_{n} \phi(y)-n P\right),
$$

up to some multiplicative constant $C=C(\phi)$.

When $\mu$ is not absolutely continuous, its distribution at some scale will be very uneven, which can be spread by (17) to all smaller scales, since constant $C$ is independent of $n$.

More specifically, if for all $k$-adic intervals $I$ one has $\mu(I) / l(I) \geq \frac{1}{2 C^{2}} \mu\left(I_{0}\right)$, then $\mu$ has a non-trivial absolutely continuous part (with density bounded away 
from zero). But measure $\mu$ is ergodic, and its absolutely continuous part is invariant, so in this case measure $\mu$ has to be absolutely continuous.

Thus if measure $\mu$ is not absolutely continuous, we can find an interval $I$ with $\mu(I) / l(I)<\frac{1}{2 C^{2}} \mu\left(I_{0}\right)$. If $I$ is of length $k^{-\ell}$, the latter means that $\mu$ is " $\left(k^{-\ell}, 1 /\left(2 C^{2}\right)\right)$ weak porous at scale zero." Taking preimages and using the equation (17), we obtain that the measure $\mu$ is $\left(k^{-\ell}, 1 / 2\right)$ weak porous, thus proving the dichotomy.

\subsection{Harmonic measure and Bourgain's dichotomy}

Harmonic measure is one of the fundamental objects in analysis, since it is related to Dirichlet problem for Laplacian, Riemann uniformization map (if $\Omega \subset \mathbb{C}$ is simply connected), equilibrium distribution for Newtonian (or logarithmic in $\mathbb{R}^{2}$ ) potential, etc.

For a domain $\Omega \subset \mathbb{R}^{d}$ and a base point $x \in \Omega$ harmonic measure $\omega_{x}$ on $\partial \Omega$ is defined as a hitting probability of Brownian motion started $x$. One needs to assume that $\Omega$ is Greenian (i.e. boundary $\partial \Omega$ is sufficiently "thick," e.g. $\operatorname{dim} \partial \Omega>d-2$ is sufficient).

Harmonic measures for different base points will be mutually absolutely continuous, so we will just write $\omega$ for harmonic measure since all geometric properties do not depend on the base point.

Compact support of $\omega$ is the whole boundary $\partial \Omega$, which might have maximal dimension and even positive volume. But $\omega$ tends to concentrate on parts of the boundary well accessible from within the domain, so one might hope to obtain some porosity-type properties.

Since compact support of $\omega$ can have positive volume, $\omega$ is not porous. One can also see that it is not porous even under weaker definition from 6.1. Divide a unit cube into $\ell=k^{d}$ cubes of equal size, then there is a domain $\Omega$ whose harmonic measure is equidistributed among these cubes. Rescaling and gluing such examples, one can construct domains $\Omega$ with "very non-porous" harmonic measure.

To produce such an example, consider domain $\Omega:=\mathbb{R}^{d} \backslash \cup_{j=1}^{\ell} B_{j}$, where $B_{j}$ are balls of radii $r_{j}$ centered at the centers of small cubes. Then $\phi:\left(r_{1}, \ldots, r_{\ell}\right) \mapsto$ $\left(\omega\left(B_{1}\right), \ldots, \omega\left(B_{\ell}\right)\right)$ is a continuous map from the tetrahedron $\left\{r_{j} \geq 0, \sum r_{j}=\right.$ $\ell\} \subset \mathbb{R}^{d}$ to the tetrahedron $\left\{\omega_{j} \geq 0, \sum \omega_{j}=1\right\} \subset \mathbb{R}^{d}$. Moreover, any face or edge of the first tetrahedron (which is given by requiring a few parameters to vanish: $\left.\left\{r_{i_{1}}=\cdots=r_{i_{k}}=0\right\}\right)$ is mapped into the corresponding face or edge of the second one (since harmonic measure of a zero radius ball vanishes). An easy topological argument shows then that the map $\phi$ is onto, thus there is a choice of radii such that $\omega\left(B_{1}\right)=\cdots=\omega\left(B_{\ell}\right)=1 / \ell$.

In the example above the harmonic measure is equidistributed among $\ell=k^{d}$ small cubes, so there is no porosity at this scale. But in most of these cubes 
the balls will be very small, $r_{j} \ll 1 / l$, and one can observe metric porosity: Hausdorff content of the support of $\omega$ in some small cube is much smaller than its size.

This was formalized by J. Bourgain in [Bou], where he proved that $\operatorname{dim}_{\mathrm{H}} \omega<$ $d-\epsilon_{d}$ for some constant $\epsilon_{d}>0$. He used the following version of porosity:

Lemma 4 (Bourgain's dichotomy, Lemma 2 in [Bou]). For any $d$ there is $\rho<d$ and large $k$ such that for every domain $\Omega$ with boundary inside the unit cube $I_{0}$ and each cube $I \subset I_{0}$ one of the following properties holds

$$
\begin{aligned}
& \text { (D) } m_{\rho}(\partial \Omega \cap I, l(I) / k)<|I|^{\rho / d}, \\
& \text { (L) } \sum_{J \in \mathcal{E}(I)} \omega(J)^{1 / 2}|J|^{1 / 2} \leq \frac{1}{10} \omega(I)^{1 / 2}|I|^{1 / 2} .
\end{aligned}
$$

Here $|I|$ denotes the volume of $I, \mathcal{E}(I)$ is the splitting of $I$ into collection of $k^{d}$ equal cubes, and $m_{\rho}(\cdot, \delta)$ is the $\rho$-dimensional Hausdorff content measured with $k$-adic cubes of sizes $\leq \delta$.

This lemma, shows that for any cube one can either obtain

(D) ( local estimate of the Hausdorff dimension of $\partial \Omega$ ), meaning that compact support of $\omega$ is metrically small (but no hole of a size $\alpha \operatorname{diam}(I)$ is guaranteed, $\partial \Omega$ might look like a very "thin," but dense "dust") or

(L) (localization of harmonic measure), which is another way to say that there is a small sub-cube in $\mathcal{E}(I)$ with disproportionally small concentration of $\omega$, just like in 6.1.

One can generalize the definition of porosity along the lines of Bourgain's dichotomy, and obtain estimates of dimension of such measures similarly to our paper. But it turns out that there are more technical complications: to obtain good estimates on the multifractal spectra of $\omega$, one has to go further, not only requiring existence of holes, but also controlling their number. For harmonic measure this was formalized by P. Jones and N. Makarov in [JM] (see also the survey paper by Makarov [Mak]).

It would be nice to have a unified definition of porosity, covering all the mentioned cases, and implying good dimension and spectra estimates. Unfortunately, it seem that such a general definition would be too technical.

\section{References}

[Bou] Jean Bourgain, On the Hausdorff dimension of harmonic measure in higher dimension. Invent. Math. 87 (1987), no. 3, 477-483

[Bow] Rufus Bowen, Equilibrium states and the ergodic theory of Anosov diffeomorphisms, Lecture Notes in Math., 470, Springer, Berlin, 1975

[EJJ] Jean-Pierre Eckmann, Esa Järvenpää, and Maarit Järvenpää, Porosities and dimensions of measures, Nonlinearity, 13 (2000), 1-18 
[F] Kenneth Falconer, Techniques in fractal geometry. John Wiley \& Sons, Ltd., Chichester, 1997

[JJ] Esa Järvenpää, Maarit Järvenpää, Porous measures on $\mathbb{R}^{n}$ : local structure and dimensional properties, preprint

[JM] Peter Jones, Nikolai Makarov, Density properties of harmonic measure, Ann. of Math. (2) 142 (1995), no. 3, 427-455

[KR] Pekka Koskela, Steffen Rohde, Hausdorff Dimension and mean porosity, Math. Ann. 309 (1997), 593-609

[Mak] Nikolai Makarov, Fine structure of harmonic measure, St. Petersburg Math. J. 10 (1999), no. 2, 217-268

[Mat] Pertti Mattila, Distribution of sets and measures along planes. J. London Math. Soc. (2) 38 (1988), no. 1, 125-132

[S] Arto Salli, On the Minkowski dimension of strongly porous fractal sets in $\mathbb{R}^{n}$, Proc. London Math. Soc. (3) 62 (1991), 353-372 